\title{
Method of 3 Dimensional Coordinate Acquisition by Combining a 2 Dimensional PSD and an Ultrasonic Sensor Unit
}

\author{
Yuya Kawahara ${ }^{\text {a, }}$, Ryuichi Udo ${ }^{\mathrm{b}}$ and Liefeng Zhang ${ }^{\mathrm{a}}$ \\ ${ }^{a}$ Kyushu Institute of Technology, 1-1 Sensui-cho Tobata-ku Kitakyushu-shi Fukuoka-ken, 804-8550, Japan \\ ${ }^{\mathrm{b}}$ Tokyo Electron Kyushu Limited, 1-1 Fukuhara Gotsu-shi Kumamoto-ken, 861-1116, Japan \\ *Corresponding Author: kawahara@boss.ecs.kyutech.ac.jp
}

\begin{abstract}
Recently, the products operated by a touch panel has been spreading. However, with multi-functionalization and advanced features of home electronics, the number of commands increased and the number of buttons has increased. Therefore, the size of buttons become smaller, the area of touch panel is limited, and the shape is limited to plate. It becomes difficult to work with. A three-dimensional touch panel may be mentioned as a method of solving this problem. Three-dimension touch panel provides a virtual touch on a space, so the size of panel and button are not dependent on the size of the device and can make any size of buttons. Moreover, intuitive operation is possible. In this paper, using a combination of two-dimensional PSD and ultrasonic sensors, we propose a method for obtaining three-dimensional coordinates of the position of the finger with high accuracy.
\end{abstract}

Keywords: PSD, ultrasonic sensor, touch panel.

\section{Introduction}

Now, the touch panel is used in various electronic devices, such as smart phone, ATM and so on. Since touch panels display the key on the display, they can arbitrarily set the size of the button and the shape within the display range. However, by this technique, the area which can be used as a touch panel is dependent on the size of equipment. Thus, for avoiding the operation mistake and for placing many buttons in the viewable area, we cannot change the size of equipment to be small.

Three-dimensional touch panel exists as a method to solve this problem ${ }^{(1)(2)}$. The three-dimensional virtual touch panel using Head Mount Display and a 3D television is displayed on space. The device acquires the three-dimensional coordinate of the finger. By this method, we can make virtual touch panel on space. Therefore, the operation range does not depend on the size of the device. Besides, we can operate it intuitively. However, the method can not be small in principle, because of triangulation.

As a conventional method, two-dimensional coordinate acquisition method exists. ${ }^{(3)}$ This uses the PSD and the ultrasonic sensor, to obtain the two-dimensional coordinates of the finger. Since the the method can detect coordinate from one point in principle, the miniaturization of the device is possible. We can make the touch panel that an operation range does not depend on equipment size. The downsizing of the device is easier than conventional techniques.

Therefore, in this study, we propose a method to detect three-dimensional coordinate using ultrasonic sensor and two-dimensional PSD. We use a modulated LED, and it is attached on a finger. The position of LED is detected PSD sensor. This method can make the device small in principle. In addition, It can detect the coordinate at high speed, because of no use of image processing.

\section{Proposed System}

\subsection{Construction of proposed system}

Figure 1 shows the configuration of this proposed system, and figure 2 shows the flowchart. In this study, since we assume that the device is operated with a finger, we should detect the position of finger. First, the user attaches the device, which is composed of red LED and ultrasonic transmission sensor, to a finger. Next, the sensing device which is composed of two-dimensional PSD and ultrasonic reception sensor is mounted to glasses, and a user 
attaches the glasses. The light of red LED is projected on the surface of two-dimensional PSD. The position of the center of gravity of the light is calculated at the PSD driver circuit. Thus, two-dimensional coordinate information is obtained. In addition, an ultrasonic wave is generated from an ultrasonic transmission element, and it is received with a supersonic wave reception element. The distance from a finger is calculated by the microcomputer using ultrasonic propagation time, and the calculated result is transmitted to a personal computer by serial communication. We can acquire the three-dimensional coordinate of the position of the finger by using both PSD and ultrasonic sensor. In addition, since the red LED is modulated, the light is easily distinguished from surrounding light. For it, we prepare band pass filter circuit.

\subsection{The Distance Measurement by Ultrasonic Sensor}

To measure the distance between the detecting device and the user's finger, an ultrasonic sensor is used. This section describes the detail of the ultrasonic wave transmitter and receiver. Figure 3 shows the xyz coordinate system. At first the user attaches a ultrasonic wave transmission element to his finger. The device sends a pulse wave made with ultrasonic oscillation circuit. A receiver is attached to the detecting device and is receives the pulse wave. The detecting device counts the ultrasonic wave arrival time with a microcomputer. In this way, it measures the distance $r$ between finger and the device.

\subsection{Dimensional Position Sensitive Detector (PSD)}

In order to measure the three-dimensional coordinates of the finger, a two-dimensional position sensitive detector (PSD) shown in Figure 4 is used.

The PSD is a kind of optical sensor using the $\mathrm{Si}$ photodiode. PSD can detect the position of the entering spot light. The feature of PSD is to detect position with high resolution and fast response.

The PSD generates the electro charge according to the amount of light reaching a light-receiving surface. The PSD has 4 terminals corresponding to the $\mathrm{X}-\mathrm{Y}$ axis. For these terminals, the resistance values from the light-receiving point to each terminal vary according to the lighted position. That is, the output currents from 4 terminals are also changing by the lighted position. By using the ration calculated by the currents from 4 terminals, the position of the light spot can be detected as the gravity of the light spot.

In this study, we use the PSD, S2044 of Hamamatsu
Photonics. Its active area is $4.7 \times 4.7 \mathrm{~mm}$.

In Figure 4, we show coordinates of a center of gravity point as $(x, y)$. According to the center-of-gravity point of this spot light, current value $I_{X 1}, I_{X 2}, I_{Y 1}$, and $I_{Y 2}$ are obtained from the electrode of the four electrodes $X 1, X 2$,

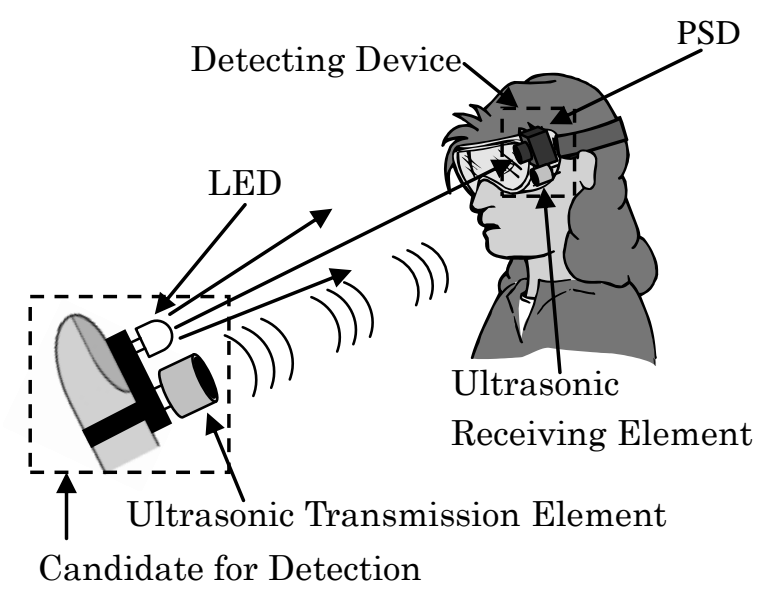

Fig. 1. System Configuration.

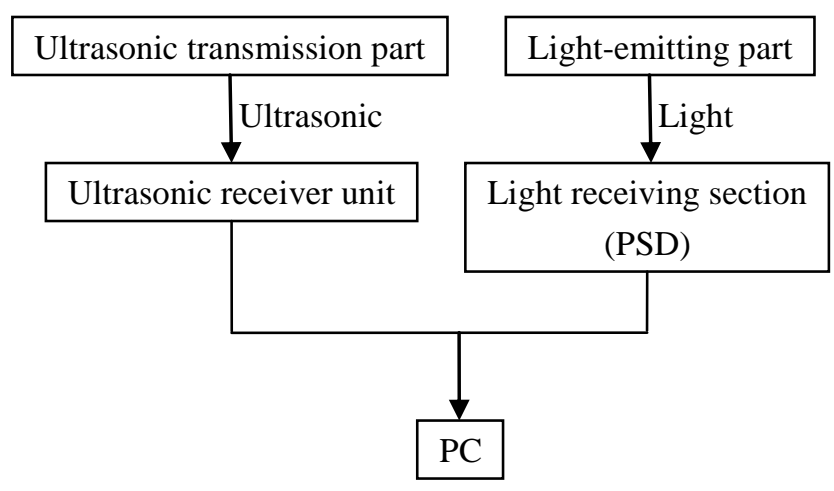

Fig. 2. Flowchart.

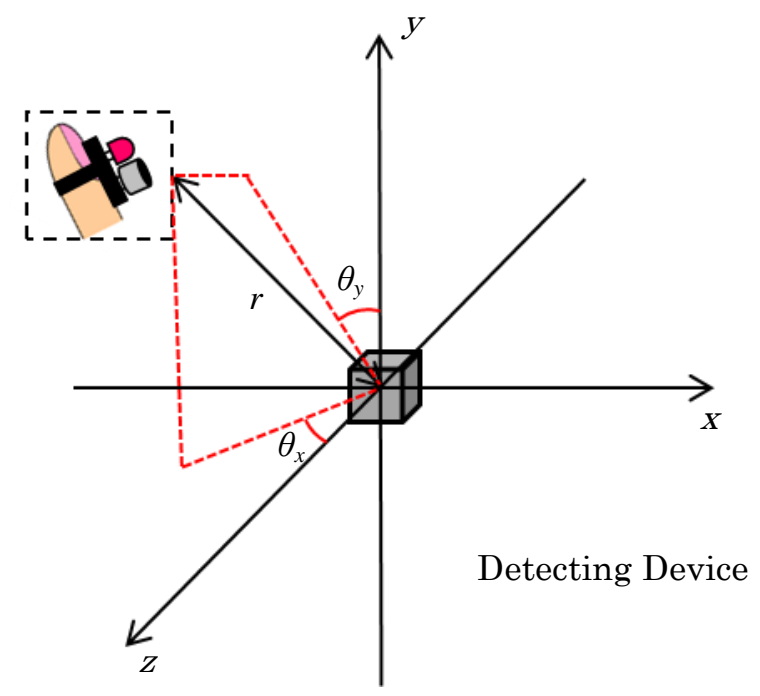

Fig. 3. XYZ Coordinate System. 
$Y 1$, and $Y 2$, respectively. In this case, the $\mathrm{X}$ coordinate of the center of gravity is the following equation.

$$
\frac{\left(I_{X 2}+I_{Y 1}\right)-\left(I_{X 1}+I_{Y 2}\right)}{I_{X 1}+I_{X 2}+I_{Y 1}+I_{Y 2}}=\frac{2 x}{L_{x}}
$$

The following equation will be the $\mathrm{Y}$ coordinate of the center of gravity.

$$
\frac{\left(I_{X 2}+I_{Y 2}\right)-\left(I_{X 1}+I_{Y 1}\right)}{I_{X 1}+I_{X 2}+I_{Y 1}+I_{Y 2}}=\frac{2 y}{L_{y}}
$$

Moreover, since $L_{X}$ and $L_{Y}$ are $L_{X}=5.7 \mathrm{~mm}$ and $L Y=5.7$ $\mathrm{mm}$ in $\mathrm{S} 2044$ respectively, the center-of-gravity of spot light can be calculated by the conversion type of equations (1) and (2).

The light of the LED on finger is focused on the surface of PSD through the lens. Figure 5 shows this relationship. Figure 6 shows OPAO'P' plane of Figure 5. Since the value $\mathrm{D}$ is constant in Figure 6 , the angle $\theta$ is calculated by the following equations. In addition, it becomes similar about the y-axis direction.

$$
\begin{aligned}
& \theta_{\mathrm{x}}=\tan ^{-1} \frac{x^{\prime}}{D} \\
& \theta_{\mathrm{y}}=\tan ^{-1} \frac{y^{\prime}}{D}
\end{aligned}
$$

\subsection{Measurement of Three-Dimensional Coordinates of the Finger}

We can calculate the $\mathrm{X}-\mathrm{Y}-\mathrm{Z}$ coordinate by using $\theta_{x}, \theta_{y}$ and $r$ as shown in the following equation.

$$
\begin{aligned}
& x=r \sin \theta_{x} \\
& y=r \sin \theta_{y} \\
& z=r \cos \theta_{y}
\end{aligned}
$$

We show a flowchart for detecting 3D coordinates in Fig. 7. We turn on LED attached on finger for a certain period. The light is received to PSD by passing through a lens. The following describes a process for extracting a modulation signal from the output signal of the PSD sensor. The I-V conversion circuit converts the current output of the PSD to the voltage. The voltage of center frequency of the LED light is extracted by using a multiple feedback type BPF.

After smoothing circuit, the output signal is incorporated into the $\mathrm{PC}$ to calculate $3 \mathrm{D}$ coordinates

\section{Experiments}

\subsection{Measurement of Distance Using Ultrasonic Sensors}

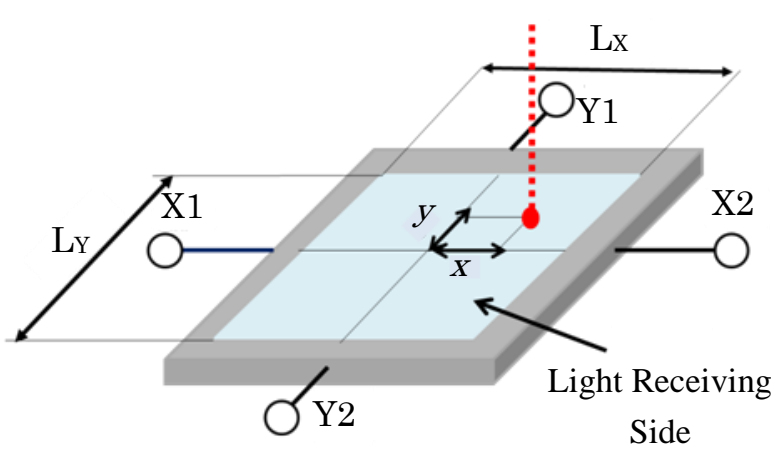

Fig. 4. 2 Dimensional PSD.

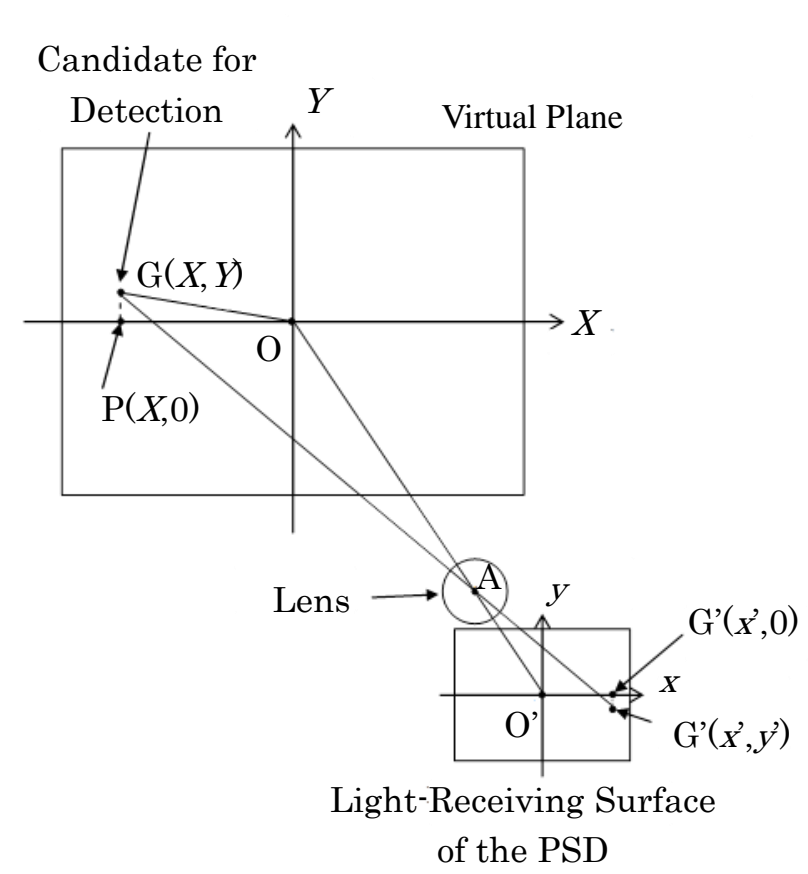

Fig. 5. State of the Light Receiving of PSD.

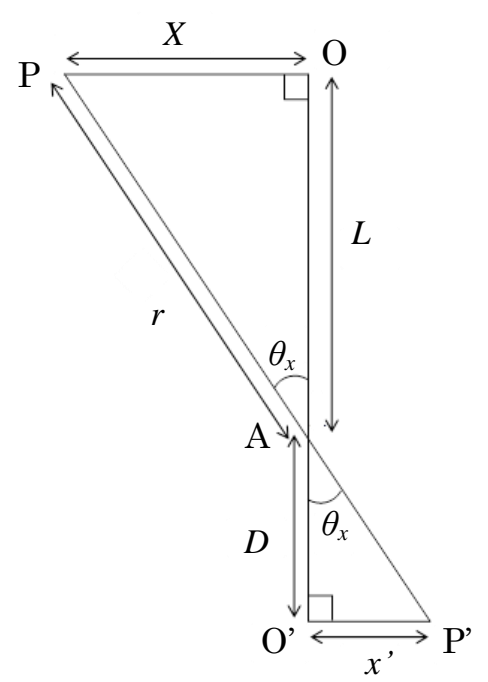

Fig. 6. OPAO'P 'plane. 
We measured the distance $r$ between finger and receiving element using an ultrasonic sensor. Figure 8 and Table 1 shows the results. From these results, we can measure the distance with error of less than $1 \mathrm{~cm}$.

\subsection{Measurement of 2D Coordinate Using PSD Sensor}

We measured the 2D coordinate of the finger using PSD sensor. When the distance $z$ is a constant, we measured $x$ and $y$ coordinate on PSD surface. Then, 2D coordinate was calculated by the equations (5) and (6). Table 2 shows the result.

\subsection{Measurement of Three-Dimensional Coordinate}

We measured the three-dimensional coordinate of the finger using PSD sensor and ultrasonic sensor. The coordinate is calculated by the equations (4), (5) and (6). Table 3 shows the result.

\section{Conclusions}

In this study, we were able to confirm that we could detect the three-dimensional coordinate of the finger using ultrasonic sensor and PSD. This method can make the device small in principle.

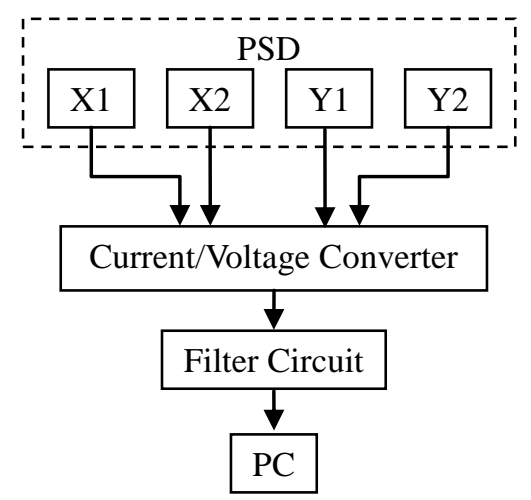

Fig. 7. Flowchart.

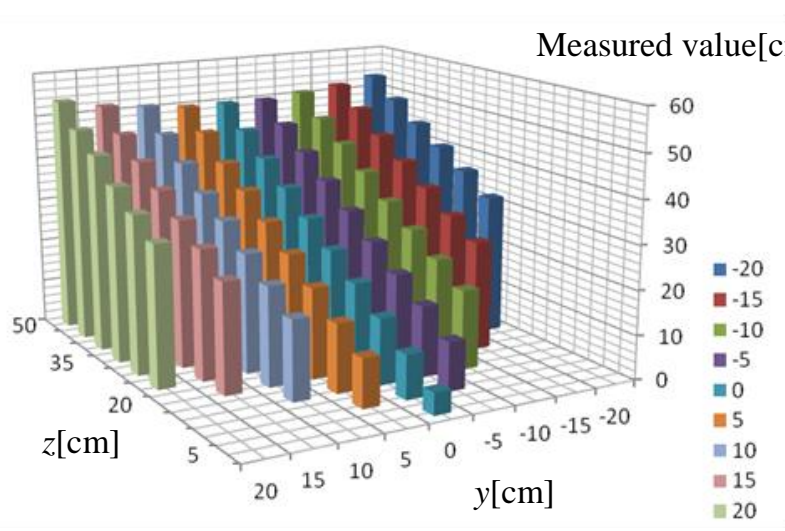

Fig. 8. OPAO'P 'plane.
Table 1. The average of the absolute error of $r$ and standard deviation.

\begin{tabular}{|c|c|c|}
\hline & $\begin{array}{c}\text { Error Mean Square } \\
{[\mathrm{cm}]}\end{array}$ & $\begin{array}{c}\text { Standard deviation } \\
{[\mathrm{cm}]}\end{array}$ \\
\hline$r$ & 0.19 & 0.18 \\
\hline
\end{tabular}

Table 2. The error mean square and standard deviation by measurement using the PSD.

(a) $\mathrm{z}=30[\mathrm{~cm}]$

\begin{tabular}{|c|c|c|}
\hline & $\begin{array}{c}\text { Error Mean Square } \\
{[\mathrm{cm}]}\end{array}$ & $\begin{array}{c}\text { Standard deviation } \\
{[\mathrm{cm}]}\end{array}$ \\
\hline$x$ & 1.43 & 1.17 \\
\hline$y$ & 1.49 & 1.25 \\
\hline
\end{tabular}

(a) $\mathrm{z}=35[\mathrm{~cm}]$

\begin{tabular}{|c|c|c|}
\hline & $\begin{array}{c}\text { Error Mean Square } \\
{[\mathrm{cm}]}\end{array}$ & $\begin{array}{c}\text { Standard deviation } \\
{[\mathrm{cm}]}\end{array}$ \\
\hline$x$ & 1.13 & 1.06 \\
\hline$y$ & 1.05 & 1.04 \\
\hline
\end{tabular}

(a) $\mathrm{z}=40[\mathrm{~cm}]$

\begin{tabular}{|c|c|c|}
\hline & $\begin{array}{c}\text { Error Mean Square } \\
{[\mathrm{cm}]}\end{array}$ & $\begin{array}{c}\text { Standard deviation } \\
{[\mathrm{cm}]}\end{array}$ \\
\hline$x$ & 1.71 & 1.60 \\
\hline$y$ & 1.87 & 1.27 \\
\hline
\end{tabular}

Table 3. The error mean square and standard deviation by measurement using the PSD and ultrasonic sensor.

\begin{tabular}{|c|c|c|}
\hline & $\begin{array}{c}\text { Error Mean Square } \\
{[\mathrm{cm}]}\end{array}$ & $\begin{array}{c}\text { Standard deviation } \\
{[\mathrm{cm}]}\end{array}$ \\
\hline$x$ & 0.96 & 0.96 \\
\hline$y$ & 1.55 & 1.08 \\
\hline$z$ & 0.20 & 0.18 \\
\hline
\end{tabular}

\section{References}

(1) Yasunaka, R : "Proposal of 3 dimension imaginary space touch panel using ultrasonic sensors", IEEE Sensors 2010

(2) Nobuyuki Furuya, Tsuyoshi Shimizu, and Akihito Ito : "Stereo Image Measuring Method Based on Projected Invariant : 3D Coordinate Calculation based on Cross Ratio and Standard Scale", Journal of the Japan Society for Precision Engineering 78(10), 875-879, 2012-10-00, Japanese

(3) Yuya Kawahara, Ryuichi Udo, and Shiyuan Yang : "Proposal of a Device Detecting Two Dimensional Coordinates Using PSD Sensor and Ultrasonic Sensor", ICIAE2013 